\title{
Using loT technologies to develop a low-cost smart medicine box
}

\author{
Danyllo V. da Silva \\ COPPE/UFRJ \\ Univ. Federal do Rio de Janeiro \\ Rio de Janeiro, Brasil \\ dvsilva@cos.ufrj.br
}

\author{
Taisa G. Gonçalves \\ COPPE/UFRJ \\ Univ. Federal do Rio de Janeiro \\ Rio de Janeiro, Brasil \\ taisa@cos.ufrj.br
}

\author{
Paulo F. Pires \\ COPPE/UFRJ \\ Univ. Federal do Rio de Janeiro \\ Rio de Janeiro, Brasil \\ paulopires@cos.ufrj.br
}

\begin{abstract}
Internet of Things (IoT) is a paradigm that has provided improvements for the day-to-day of society. This paradigm has been applied in several domains such as ambient assisted living (AAL), energy, transportation, environmental, urban monitoring, and healthcare. In the healthcare domain, IoT offers many advantages, such as enable continuous health monitoring, improve quality life and comfort, among others. A kind of IoT application in this domain is smart medicine box, a device that provides medicines treatment monitoring of users. It allows health professionals to verify users' treatment compliance and supports decision-making. Most of the smart medicine box projects found in the literature are still expensive and do not address some characteristics of IoT systems such as scalability, latency, time to response, among others. Taking into account this scenario, this work proposes a low-cost IoT system prototype to support users during their medicines manipulation. The proposal employs edge-computing concept to add intermediate layer improving the communication among devices and services.
\end{abstract}

\section{KEYWORDS}

Internet of Things (IoT), Healthcare, e-health, Smart medicine box, Web application

\section{Introduction}

The technological evolution generated new paradigms, such as the Internet of Things (IoT) [1] [2]. IoT has been recognized as a revolution in information and communication technology (ICT), and its adoption offers opportunities for many domains and companies [1] [3]. In the IoT paradigm every object can be identified, addressable, controlled, and monitored through the internet [1] [2][4] [5] These objects can communicate and collaborate with others to perform tasks to benefit end-users [5].

In this sense, IoT allows the connection of smart objects such

In: XVIII Workshop de Ferramentas e Aplicações (WFA 2019), Rio de Janeiro, Brasil. Anais Estendidos do Simpósio Brasileiro de Sistemas Multimídia e Web (WebMedia). Porto Alegre: Sociedade Brasileira de Computação, 2019. (c)2019 SBC - Sociedade Brasileira de Computação.

ISSN: 2596-1683 as sensors, actuators, and other devices to the internet bringing a considerable impact on human society [1] [5] [6]. This paradigm has been employed in several domains, such as ambient assisted living (AAL), energy, transportation, environmental, urban monitoring, and healthcare [5] [6].

One of the application domains that benefit from IoT is Healthcare (or e-health). IoT systems in e-health are used to monitor patients' vital signs through body sensors, electronic devices, and information and communication technologies. These systems assist doctors, patients, relatives, and healthcare centers in remote assistance [2] [5] [7], and relevant healthcare information can be collected and managed by them [1].

In addition, several advantages can be noted with the use of e-health systems such as, quality improvement of the treatments and diagnostics [3] [5], support on decision-making [5], monitoring of the patients' conditions and vital signs [3] [5], improving health and well-being [2], faster detection of emergency situations [5], among others.

A particular application of e-health is the smart medicine box [1] [3] [8] [9] [10] [11] [12]. They focus on problems related to medicines management. In some scenarios, they help to detect if the patient follows the treatment or abuses of the prescription that have been done [3] [12]. To solve this problem, the medicine box solution aims to remind patients about their prescribed medicines. It alerts the patients to take their medication at the right time and right proportion [3] [9] [12].

Smart medicine box generally notifies users about their medicines using smartphone notifications, alarm sound, a visual indicator (LEDs), among others [9] [12]. In general, these boxes present functionalities such as: treatment monitoring [10], noncompliance management [3] [10], health monitoring and diagnostics [9] [10], routine remote monitoring [9], online prescription [3], emergency support [10], pharmacists notification about medicine refill [12], among others. Furthermore, healthcare professionals and pharmacist can have a view about patients' treatment and follow if the prescribed treatment is done correctly [3] [12]. Moreover, some services are essential for IoT system's operation such as interoperability [1] [3], scalability [9], performance [1] [3] [10], data processing [1] [3] [10], and security [1] [3] [10].

These solutions are still expensive, which hinders the broad access to this technology. Therefore, this work proposes the development of a low-cost smart medicine box prototype that 
aims to remind its users to take the medicines at the right time, following the schedule previously configured on the user interface. Edge computing concept was employed to provide a robust architecture to achieve scalability, performance, interoperability, security, among others. This prototype is an initial solution with reduced scope to be used as a proof of concept.

Besides, this section that defines IoT and gives a short overview of the problem here addressed, this work is organized as follows. Section 2 presents related researches about smart medicine box. Section 3 presents the proposed solution detailing the system requirements. The architecture is presented in section 4, showing how the proposed solution addresses the requirements. Finally, in section 5, the conclusion is presented, and future researches are discussed.

\section{Related work}

In this section, we present a brief discussion on the smart box solutions available in the literature.

Rosli and Husaini [8] proposed a smart medicine box to improve the autonomy of introvert patients. The increasing number of patients in the hospitals leads to surplus nurses' workload. Based on this scenario, the authors developed an IoT device and applications to support nurses during their activities. This device helps nurses in the advice and monitoring of medicines treatment of introvert patients. A proof of concept involving device and apps development was performed. The authors highlighted the usefulness of the developed device to facilitate the nurses' job and provide comfort to patients.

Pang et al. [1] [10] and Yang et al. [3] proposed a health-IoT solution composed of devices and services through an integrated architecture. This system supports doctors to prescribe medicines and monitor users' in their treatments. It offers some actions such as to send alarm messages, play an audio clip, and generate vibration. Furthermore, this solution includes the implementation of a wearable device to monitor users' vital signals. The data collected can be accomplished remotely and through the smart medicine box LCD. One authentication is implemented to identify the user's access. The authors evaluated the solution regarding performance analysis and feedbacks that show the feasibility of the system.

Srinivas et al. [9] proposed an IoT healthcare platform composed of a smart medicine box to monitor patients' health. The device gives alerts (smartphone notifications, indicator light (LEDs), an audible alert - buzzer) allowing patients to take their medicines at the right time; and it updates the information about medicines in the patient's smartphone. Doctors can monitor the routine details of the patients and prescribe the medication through a webpage.

Najeeb et al. [12] proposed an IoT system to solve the problem of patients that forget or consume medicines out of recommended by the doctor. This system helps nurses in the prescription of medicines at the right time and proportion. The system presents functionalities such as medicines prescription, patients' access control, medicines consumption monitoring for pharmacists and doctors, a tracking system for medicine refill, among others.

Zhang et al. [11] designed an intelligent medicine box monitoring and management system. The system applies IoT technology to remind patients about medicines manipulation. This device enables patients to visualize relevant information on the touch screen as medicines' name, medicines' dose and time, notes, among others. The proposed solution includes functionalities such as configuration, monitoring, log management, among others.

Analyzing the presented researches, some points can be observed. First, some solutions [1] [3] [10] [12] involve expensive devices such as body sensors, medicine package, among others, increasing the solution cost. Second, some of them present a limitation in terms of scalability and flexibility regarding devices, services and applications [8] [11] [12]. Third, some systems [11] [12] do not present solution to deal with latency, response time, and data processing.

For these reasons, this work proposes a low-cost smart medicine box employing edge architecture. The solution employs only low-cost and easily accessible technologies (both hardware and software) facilitating its use in any community where budget is a concern. Moreover, the proposal is based on a robust architecture that facilitates the implementation of useful services such as security, interoperability, scalability, performance, data processing, data storage, in a distributed fashion. The proposed architecture also provides decoupling among devices and applications and the system's life cycle management to facilitate system operation and maintenance. In the next sections, we describe the proposed solution, its functions, and the technologies employed to achieve desired functionalities.

\section{Proposed solution}

The work presented in this paper proposes a low-cost smart medicine box intend to remind users to take their medicines at the right time. In order to define the system requirements an adhoc literature review was performed [1] [3] [8] [9] [10] [11] [12]. Then, the functionalities were identified and transformed into IoT scenarios. Besides that, non-functional requirements were identified to satisfy the characteristics of IoT systems.

Regarding IoT scenarios, we applied a scenario-based technique named ScenarIoT [13]. The scenario can be defined as a sequence of actions or an ordered set of interactions among parts [13]. We choose ScenarIoT because it can be employed during requirements specification, architecture definition, documentation activities, and system's features idealization. This technique supports analysts during early development activities and suggests a list of IoT arrangements with their information catalogs. The IoT arrangements are graphical representation of the IoT system elements representing interactions among them. We provide a brief detail of the requirements developed in our solution. They are categorized in IoT scenarios and nonfunctional requirements. 
a) IoT scenarios

1. Schedule times to remind the user to take the medicines - set the times those users must take their medicines through a web interface. Then, the smart medicine box will alarm at the scheduled time. The arrangement associated to this scenario is IIA-6: Actuation triggered by a software system, based on no-IoT data (see Figure 1).

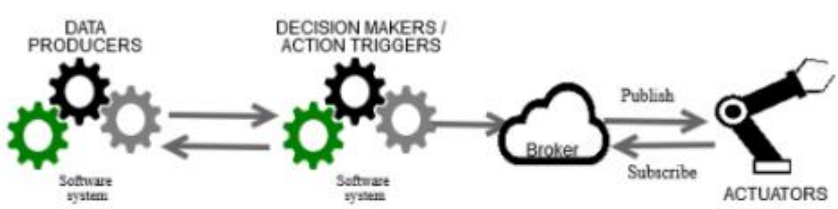

Figure 1: IIA-6 arrangement [13]

2. Visualize medicine box state and data - through a web interface, users can visualize alarms configuration, box state, and slots status. This function enables health professionals, caregivers, and family, among others to monitor and manage medicines consumption. The IIA-1: Data exhibition (see Figure 2) is the arrangement associated to this scenario.

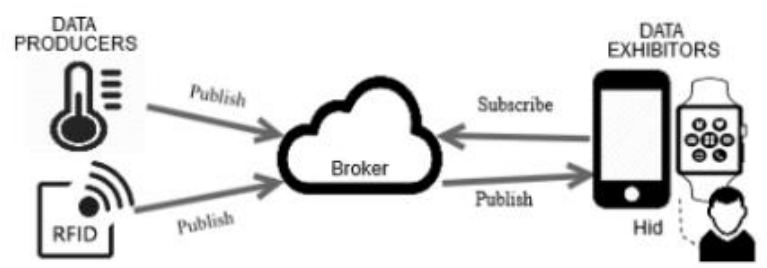

Figure 2: IIA-1 arrangement [13]

3. Remember users to take their medicines through alarms - draw users' attention through visual indicator and alarm sound to remind them to take medicines. The arrangement associated to this scenario is IIA-3: Actuation triggered by a software system (see Figure 3).

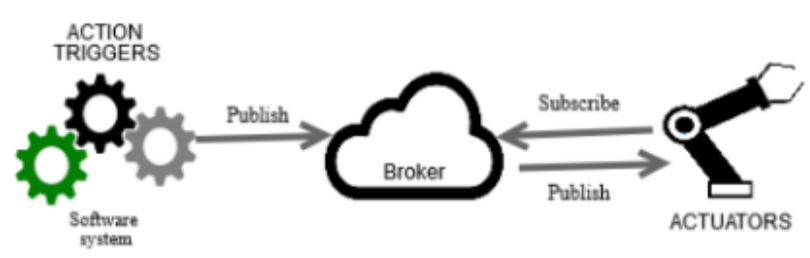

Figure 3: IIA-3 arrangement [13]

\section{b) Non-functional requirements}

1. Scalability - implement architecture to include a high number of devices and applications without impact in the current operation.
2. Decoupling among devices and applications - enable interactions among devices and applications without previous knowledge about each other [7].

3. Data processing - provide local data compression, data fusion, and data filtering for better resource usage.

4. Data storage - provide cloud architecture for data storage.

5. Performance - permit low latency communication and better response time among systems' components.

6. Security - enable access control management among devices and data.

7. Interoperability - provide interoperability among devices, protocols, and data. This function enables communication between heterogeneous devices from several manufacturers with different communication interfaces.

\section{Smart medicine box Architecture}

Considering IoT systems nature, several challenges need to be addressed. A large number of devices demands new technological solutions regarding several IoT characteristics such as interoperability, scalability, latency, among others. To deal with some of these characteristics, the fog-computing concept can be employed.

Fog computing, also called edge computing, is a paradigm based on a hierarchical system architecture that incorporates an intermediate layer between the cloud and end-devices [6]. The fog layer complements cloud-computing services by adding a gateway. Thus, additional services are provided, such as energyefficiency, performance, reliability, interoperability, mobility, load balancing, efficient scalability, and low-latency response [2] [6]. This layer also facilitates the communication between sensors layer and cloud layer [6].

A fog-based IoT architecture is composed of three layers, as shown in Figure 4. Smart devices that are equipped with identification, sensing, or actuation behaviors build the smart devices layer. The edge/fog layer consists of distributed gateways that provide several services such as data aggregation, filtering, and dimensionality. Last, the cloud layer provides a cloud-computing platform that offers a highly scalable and robust environment for IoT systems. The cloud layer provides services such as broadcasting, data warehouse, data analytics, user interfaces, among others.

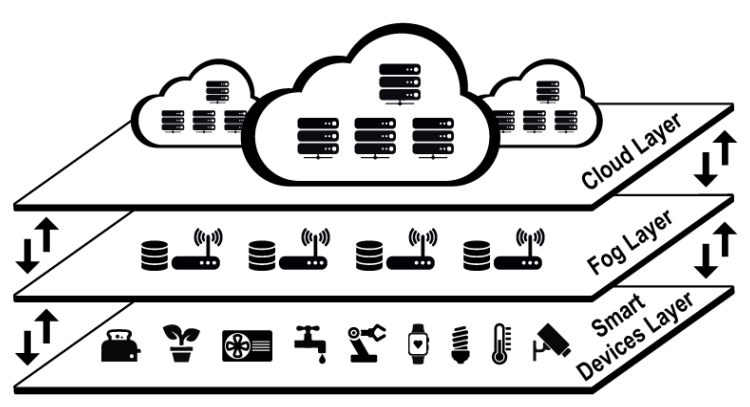

Figure 4: Generic fog-based IoT architecture (adapted from [6]) 
To achieve the desired goal, the architecture proposed in this work adopts the fog-based IoT architecture to provide a robust and scalable solution. The architecture involves the following components: smart devices network, distributed gateways, cloud server, and web clients.

\section{a) Smart devices network}

The smart devices available in users' homes or hospitals form local networks of devices. These devices are equipped with identification, sensing, and communication capacity to provide useful services for users. The proposed solution involves only one device named smart medicine box that is the core of the system, and it is responsible for reminding users about their medicines. This component addresses the IoT scenarios 2 and 3 . It provides the status of the box and medicines slots. Furthermore, this device performs alarms and visual indications to remind users to take medicines.

The box has four slots where each one represents one moment of the day. When it is the moment (scheduled time) to take medicines, the smart medicine box turns on the respective LED, and the buzzer beeps to remind the user to take their pills. The following items were employed to build this device (see Figure 5): 1 microcontroller esp8266; 1 multi-use purpose box; 4 LEDs (different colors); 1 buzzer; and 1 tilt sensor.

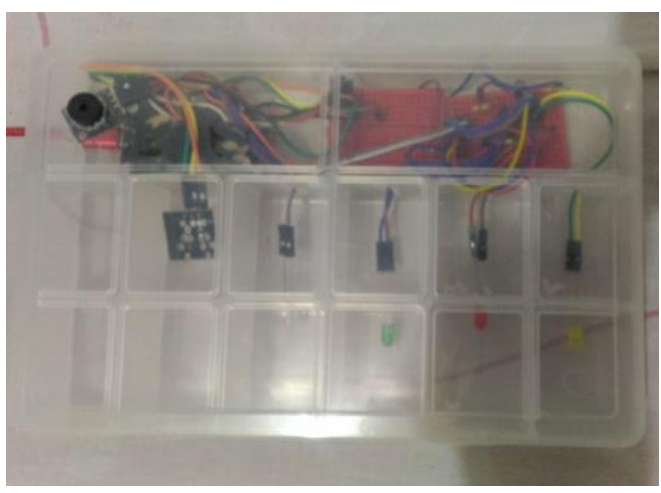

Figure 5: Smart medicine box prototype ${ }^{1}$

\section{b) Distributed gateways}

This component is responsible for managing the connection between the smart medicine box and cloud services. Gateways provide functions such as communication management, firewall, access control, lifecycle device management, mobility, load balancing, scalability, low-latency response, energy efficiency, interoperability, and reliability, among others. Gateways also can act as a router or access point. The technologies used in this component were:

- $\quad$ Raspberry Pi model 3;

- Eclipse Kura gateway for the Internet of Things;
- $\quad$ MQTT (Message Queuing Telemetry Transport) protocol.

This component addresses the IoT scenario 1 , and it is responsible for managing the schedules and sending messages to the medicine box, which handles alarms. The technical requirements addressed are: scalability, decoupling among devices and applications, data processing, security, interoperability, performance, and life cycle management.

\section{c) Cloud server}

This component provides several functions as scalability, availability, performance, robustness, reliability, security, ondemand resource usage, data storage, data analysis, access management, device management, among other advantages. The technologies used to provide these services were:

- Google Virtual Machine;

- $\quad$ Eclipse Kapua IoT cloud platform (see Figure 6).

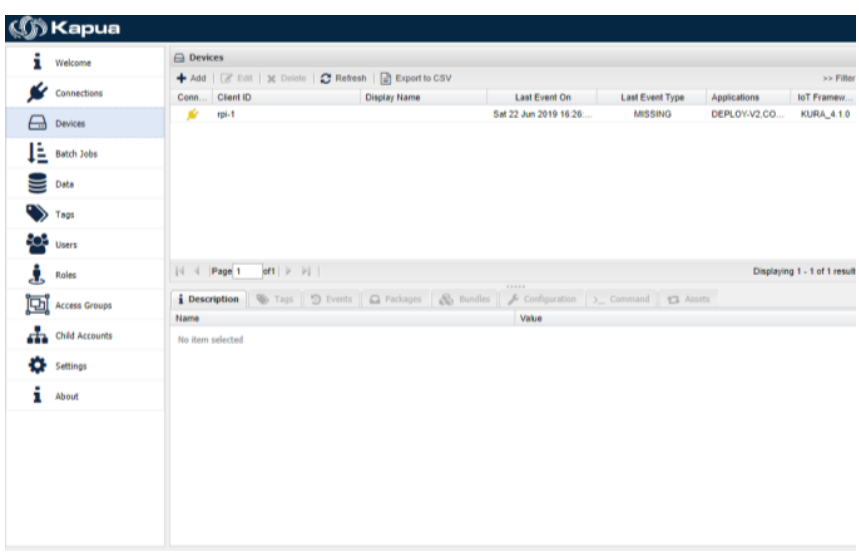

Figure 6: Kapua dashboard

This component addresses the technical requirements such as scalability, decoupling among devices and applications, data processing, data storage, performance, security, and interoperability.

\section{d) Web clients}

This component provides the user interface (see Figure 7) that exhibits information about the box state and the four slots. Furthermore, users can configure the schedule time of their medicines. NodefS was used to implement the user interface and Heroku to provide the application lifecycle management. This component addresses the IoT scenarios 1 and 2, providing the user interface. With the interface, we can configure the alarms and enable data visualization about the configuration, box state, and slots status.

\footnotetext{
${ }^{1}$ Demonstration available on: https://www.youtube.com/watch?v=5EzHixZK4YE
} 


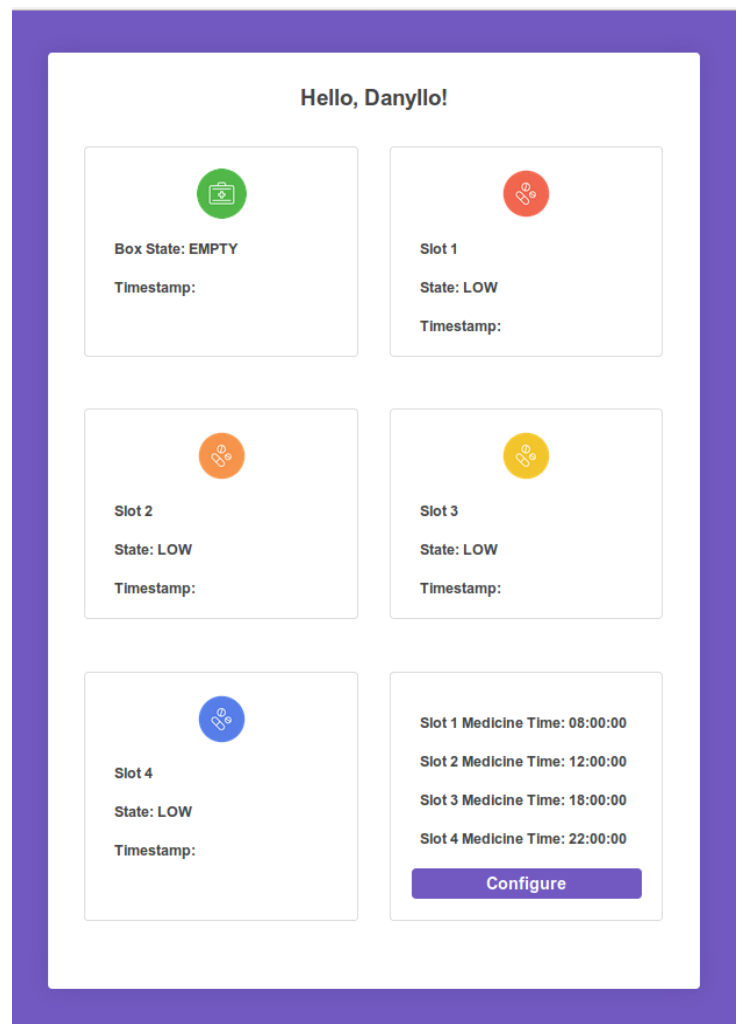

Figure 7: User interface

\section{Conclusion and future works}

IoT systems offer several advantages for our daily life, providing comfort, and improving life quality. This paradigm has been adopted in various domains, such as healthcare. In such domain, a common problem is the compliance of users' medicines consumption addressed by some authors [1] [3] [8] [9] [10] [11] [12]. These solutions use IoT technologies to solve this problem.

This work proposed a low-cost smart medicine box system employing a robust architecture to support users and health professionals during medicines consumption. The solution is still a proof-of-concept developed in the context of a master's course at COPPE/UFRJ and needs to be improved and evaluated. The initial perspective was to employ cutting-edge technologies learned during this course. As a result, we identified that this solution could be an agent to improve the comfort and quality of life.

The proposed architecture enables to embody other types of devices such as wearable, electronic devices, home appliances, among others, offering infinite possibilities of applications and functions. This system can be improved, providing a more flexible way to schedule medicines consumption alarms such as twice a week, three times a week, every other day, among others.

Another limitation is the users' activity detection related to medicines consumption. The Tilt sensor only detects if the box was opened or closed and did not detect if the user takes the medicines. About energy consumption, this box depends of the wired energy cable. A feasible solution could be to employ a battery as an energy source. Another improvement is to implement data analysis functionality to support health professionals in the identification of disease advancement, change of user behavior, treatment effect, among others.

Future work comprises to incorporate the features previously mentioned in the solution here presented, to build a complete AAL to benefit aged people and users with health problems or chronic diseases. Additional services may be included, such as health activity monitoring, fall detection, and others. An evaluation is also required to collect users' feedbacks and to improve the system's operations and functionalities.

\section{ACKNOWLEDGMENTS}

Danyllo Silva acknowledges COPPEAD/UFRJ for the support to conduct the master course. Taisa Gonçalves acknowledges CNPq for the grant (154004/2018-9) of a postdoctoral scholarship.

\section{REFERENCES}

[1] Z. Pang, L. Zheng, J. Tian, S. Kao-Walter, E. Dubrova, e Q. Chen, "Design of a terminal solution for integration of in-home health care devices and services towards the Internet-of-Things", Enterprise Information Systems, vol. 9, $\mathrm{n}^{\mathrm{o}} 1$, p. 86-116, 2015.

[2] A. M. Rahmani et al., "Smart e-Health Gateway: Bringing intelligence to Internet-of-Things based ubiquitous healthcare systems", in Proceedings of the 12th Annual IEEE Consumer Communications and Networking Conference, 2015, p. 826-834.

[3] G. Yang et al., "A Health-IoT Platform Based on the Integration of Intelligent Packaging, Unobtrusive Bio-Sensor, and Intelligent Medicine Box", IEEE Transactions on Industrial Informatics, vol. 10, no 4, p. 2180-2191, 2014.

[4] K. Paridel, E. Bainomugisha, Y. Vanrompay, Y. Berbers, e W. De Meuter, "Middleware for the Internet of Things, Design Goals and Challenges", Electronic Communications of the EASST, vol. 28, p. 392-364, 2010.

[5] P. Maia et al., "A Web Platform for Interconnecting Body Sensors and Improving Health Care”, Procedia Computer Science, vol. 40, p. 135-142, 2014.

[6] A. M. Rahmani et al., "Exploiting smart e-Health gateways at the edge of healthcare Internet-of-Things: A fog computing approach", Future Generation Computer Systems, vol. 78, p. 641-658, 2018.

[7] P. Maia, A. Baffa, E. Cavalcante, F. C. Delicato, T. Batista, e P. F. Pires, "A Middleware Platform for Integrating Devices and Developing Applications in E-Health", in Proceedings of the XXXIII Brazilian Symposium on Computer Networks and Distributed Systems, 2015, p. 10-18.

[8] E. Rosli e Y. Husaini, "Design and Development of Smart Medicine Box", IOP Conference Series: Materials Science and Engineering, vol. 341, p. Paper 012004, 2018.

[9] M. Srinivas, P. Durgaprasadarao, e V. N. P. Raj, "Intelligent medicine box for medication management using IoT", in Proceedings of the 2nd International Conference on Inventive Systems and Control, 2018, p. 32-34.

[10] Z. Pang, J. Tian, e Q. Chen, "Intelligent packaging and intelligent medicine box for medication management towards the Internet-of-Things", in Proceedings of the 16th International Conference on Advanced Communication Technology, 2014, p. 352-360.

[11] T. Zhang, Z. Liu, X. Cao, e W. Xia, "Intelligent Medicine Box Monitoring and Management System", in Proceedings of the International Conference on Computer Science and Mechanical Automation, Hangzhou, China, 2015, p. 153-156.

[12] P. K. Nijiya Jabin Najeeb, A. Rimna, K. P. Safa, M. Silvana, e T. K. Adarsh, "Pill Care-The Smart Pill Box with Remind, Authenticate and Confirmation Function", in Proceedings of the International Conference on Emerging Trends and Innovations In Engineering And Technological Research, Ernakulam, 2018, p. $1-5$.

[13] V. M. Silva, "SCENARIoT Support for Scenario Specification of Internet of Things-Based Software Systems", Master's Dissertation, Federal University of Rio de Janeiro, Rio de Janeiro, 2019. 